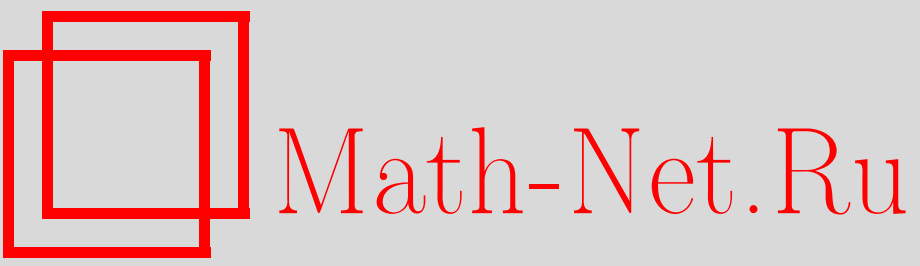

Д. Ю. Ирз, В. Н. Рыжов, Е. Е. Тареева, Статистическая механика системы вихрей в тонкой сверхпроводящей пленке в кольцевом приближении III. Учет конечного размера ядра вихря, ТМФ, 1996, том 107, номер 1, 100-114

DOI: https://doi.org/10.4213/tmf1141

Использование Общероссийского математического портала Math-Net.Ru подразумевает, что вы прочитали и согласны с пользовательским соглашением

http: //www.mathnet.ru/rus/agreement

Параметры загрузки:

IP: 34.229 .108 .108

26 апреля 2023 г., 10:16:39 
ТЕОРЕТИЧЕСКАЯ

И МАТЕМАТИЧЕСКАЯ

ФИЗИКА

Том 107, № 1

апрель, 1996

Д. Ю. Ирз, В. Н. Рыжов, Е. Е. Тареева

\section{СТАТИСТИЧЕСКАЯ МЕХАНИКА СИСТЕМЫ ВИХРЕЙ В ТОНКОЙ СВЕРХПРОВОДЯЩЕЙ ПЛЕНКЕ В КОЛЬЦЕВОМ ПРИБЛИЖЕНИИ III. УЧЕТ КОНЕЧНОГО РАЗМЕРА ЯДРА ВИХРЯ}

Статистическая механика системы вихрей в двумерном сверхпроводнике построена в рамках кольцевого приближения с учетом конечности размера ядра вихря. Показано, что при больших энергиях ядра вихря диссоциация пар вихрь-антивихрь происходит непрерывным образом, а при малых энергиях ядра - посредством перехода первого рода.

1. В работах $[1,2,3]$ была построена статистическая механика системы вихрей в тонкой сверхпроводящей пленке с помошью приближения, аналогичного приближению Дебая для кулоновской плазмы. При этом вихри рассматривались как точечные, т.е. размер и пространственная протяженность ядра вихря не были учтены явно. При этом радиус ядра вошел в теорию только косвенным образом - через энергию изолированного вихря $\varepsilon_{1}$. Из вириального разложения было получено уравнение для плотности свободных вихрей. Это уравнение позволило описать как поведение типа Костерлица-Таулесса $(\mathrm{KT})[4,5]$ в окрестности соответствуюшей температуры перехода $T_{\mathrm{KT}}$ (плавный, но очень быстрый кроссовер от состояния с низкой плотностью свободных вихрей к состоянию с высокой плотностью), так и хвост электросопротивления, продолжающийся в область низких температур. Было показано также, что во внешнем магнитном поле $H_{0}$ при условии, что $H_{0}$ превышает некоторое критическое значение, при температурах меньших $T_{\mathrm{KT}}$ имеет место скачкообразный рост плотности свободных вихрей, приводяший соответственно к росту сопротивления.

Предположение о том, что вихри точечные, является одним из основных приближений, использовавшихся в работах $[1,2,3]$. Кроме того, в этих работах не учитывались пиннинг вихрей и конечная толшина пленки. В дальнейшем конечная толшина пленки и изгиб вихрей были учтены в работе [6]. В этой работе было показано, что взаимодействие вихрей в тонкой пленке конечной толшины отличается от хорошо известного результата [7] для бесконечно тонкой пленки новой эффективной глубиной проникновения

$$
e \Lambda^{\prime}=\lambda \operatorname{cth} \frac{d}{2 \lambda} .
$$

Хотя внесение этой поправки в конкретные расчеты $[1,2,3]$ дает малый эффект, однако для большей толшины пленки и более низких температур эффект может быть значи- 
тельным. Кроме того, в работе [6] был проведен учет (в главном порядке по толщине пленки) изгиба вихрей в духе квазиклассического приближения. Эта поправка оказалась несущественной для разумных значений констант во всем температурном диапазоне. В работе [6], так же как в работах $[1,2,3]$, вихри считались точечными.

В работе [3] была также изучена зависимость поведения системы от энергии ядра вихря $E_{c}$, связанной с радиусом ядра $r_{0}$, и было показано, что при $r_{0}$ (и соответственно энергии ядра), меньшем некоторого критического значения $r_{0}^{c}$, поведение системы изменяется кардинальным образом: непрерывная кривая, описываюшая зависимость плотности вихрей от температуры, становится разрывной, при этом анализ поведения энергии системы позволил интерпретировать поведение плотности как переход первого рода. Вместе с тем полученное решение обладает рядом нефизических черт. В частности, имеется конечная область температур, в которой решение не сушествует. Кроме того, в окрестности $T_{\mathrm{KT}}$ плотность вихрей обрашается в бесконечность. Подобное поведение было объяснено тем, что вихри рассматриваются как точечные, и было предположено, что учет конечности размера ядра вихря может исправить указанные недостатки.

В настояшей работе мы делаем попытку учесть неточечность вихря и конкретную форму распределения "заряда" в вихре и изучить ее влияние на вид потенциала взаимодействия вихрей. Эти результаты используются затем для построения свободной энергии Гиббса и уравнения для плотности свободных вихрей, т.е. в конечном счете для построения фазовой диаграммы системы вихрей в тонкой сверхпроводящей пленке и определения кривой электросопротивления. Полученные ранее результаты существенно не меняются при низкой плотности вихрей, когда перекрывания плотностей не имеют места. Вместе с тем удалось разрешить трудности (см. раздел V работы [3]), связанные с нефизическими решениями полученных там уравнений: оказалось, что существует область значений параметров, когда фазовый переход может стать фазовым переходом первого рода.

Работа построена следующим образом. В разделе 2 на основе исследования уравнений Гинзбурга-Ландау (ГЛ) для двумерной пленки изучается влияние формы волновой функции ГЛ $\psi$ (связанной со структурой вихря) на потенциал взаимодействия вихрей. Для этого потенциала выводится достаточно общее выражение (22), которое в пределе точечных вихрей переходит в хорошо известный результат Пирла [7]. В разделе 3 проводится анализ полученного выражения (22), которое в общем случае является весьма громоздким. На основе точных результатов (получение которых вынесено в приложение) предлагается упрошенный модельный потенциал, который и используется дальше. Далее, в разделе 4 в духе работ $[1,2,3]$ строится статистическая механика системы вихрей с полученным новым взаимодействием, находится свободная энергия и выводится уравнение для плотности свободных вихрей. В разделе 5 обсуждаются решения полученных уравнений при различных значениях параметров. Оказалось, что учет "размазывания" вихрей приводит к возможности кроссовера от перехода типа КT к переходу первого рода при изменении условного размера вихря.

2. Напомним, что выражение для потенциала взаимодействия точечных вихрей в бесконечно тонкой сверхпроводяшей пленке было получено в работе [7] и имеет вид

$$
\Phi_{i j}\left(r_{i j}\right)=\frac{s_{i} s_{j} \varphi_{0}^{2}}{8 \pi \Lambda}\left[H_{0}\left(\frac{r_{i j}}{\Lambda}\right)-N_{0}\left(\frac{r_{i j}}{\Lambda}\right)\right]
$$




$$
\begin{aligned}
& \Phi_{i j}\left(r_{i j}\right) \approx-\frac{s_{i} s_{j} \varphi_{0}^{2}}{4 \pi^{2} \Lambda} \ln \left(\frac{r_{i j}}{\Lambda}\right), \quad r_{i j} \ll \Lambda, \\
& \Phi_{i j}\left(r_{i j}\right) \approx \frac{s_{i} s_{j} \varphi_{0}^{2}}{4 \pi^{2} r_{i j}}, \quad r_{i j} \gg \Lambda .
\end{aligned}
$$

Здесь $H_{0}(x)$ - функция Струве, а $N_{0}(x)$ - функция Неймана; $r_{i j}$ - расстояние между центрами вихрей; $\Lambda$ - эффективная двумерная глубина проникновения, связанная с глубиной проникновения $\lambda$ для массивного сверхпроводника и толщиной пленки $d$ соотношением $\Lambda=\frac{2 \lambda^{2}}{d}$, а $s_{i}= \pm 1$ - “заряд” вихря, определяемый направлением в нем магнитного поля. Если мы хотим учесть конечную толшину пленки, мы должны использовать в качестве эффективной двумерной глубины проникновения величину (1).

Наша первая задача - определить, как изменится выражение (2) при учете конкретной формы распределения заряда неточечного вихря. Для этого рассмотрим уравнения гЛ $[8,9]$

$$
\begin{gathered}
\left(i \nabla^{\prime}+\frac{\mathbf{a}}{\xi^{\prime} \sqrt{2}}\right)^{2} \psi=\frac{1}{\xi^{\prime 2}} \psi\left(1-|\psi|^{2}\right), \\
\nabla^{\prime 2} \mathbf{a}=\left\{\frac{i \xi^{\prime}}{\sqrt{2}}\left(\psi^{*} \nabla^{\prime} \psi-\psi \nabla^{\prime} \psi^{*}\right)+|\psi|^{2} \mathbf{a}\right\} .
\end{gathered}
$$

Здесь использована так назьваемая приведенная система единиц, в которой все длины измеряются в единицах глубины проникновения $\lambda$, магнитные поля - в единицах критического поля $H_{c}$, а $\psi(\mathbf{r}, z)=f(\mathbf{r}, z) e^{i \phi(\mathbf{r}, z)}-$ волновая функция, нормированная таким образом, что $|\psi|^{2}=1$ внутри сверхпроводяшей фазы, $\mathbf{r}$ - двумерный радиус-вектор в плоскости $(x, y), \xi^{\prime}$ - длина когерентности (также измеряемая в единицах $\lambda$ ), т.е. обратный параметр ГЛ. Энергия измеряется в единицах $\frac{\lambda^{3} H_{c}{ }^{2}}{4 \pi}$. $\nabla^{\prime}$ - оператор дифференцирования в трехмерном пространстве $(\mathbf{r} / \lambda, z / \lambda)$. В дальнейшем, пока это не будет особо оговорено, мы не будем помечать величины в приведенной системе единиц штрихом. В этих единицах свободная энергия ГЛ принимает вид

$$
F_{\text {Гл }}=\int d^{3} x\left\{\frac{\mathbf{b}^{2}}{2}+\xi^{2}\left|\left(\nabla-\frac{i \mathbf{a}}{\sqrt{2} \xi}\right) \psi\right|^{2}+\frac{1}{2}\left(|\psi|^{2}-1\right)^{2}\right\} .
$$

Здесь $\mathbf{b}=\nabla \times \mathbf{a}-$ магнитное поле, а интегрирование ведется по всему трехмерному пространству $\mathbf{x}=(\mathbf{r} / \lambda, z / \lambda)$. Кроме того, мы опустили несущественную для нас свободную энергию нормального состояния.

Уравнения (3), (4) связаны друг с другом, но тем не менее в некотором смысле можно сказать,что за вид $\psi$ отвечает уравнение (3), а за форму вектор-потенциала $\mathbf{a}$ - уравнение (4). Стандартный подход к решению этих уравнений заключается в том, что вместо решения уравнения (3) делаются некоторые предположения о структуре функции $\psi$ в случае изолированного вихря, после чего для вычисления энергии взаимодействия вихрей принимается так называемое суперпозиционное приближение. Его суть заключается в том, что ток каждого вихря считается зависяшим только от структуры этого вихря, но не от положения других вихрей ${ }^{1)}$. Это предположение справедливо, если вихри расположены достаточно далеко друг от друга (т.е. $r \gg \lambda$ ).В нашем же случае необходимо

\footnotetext{
1) Здесь под структурой вихря мы понимаем вид волновой функции $\psi(\mathbf{r})$.
} 
учесть и область $r \lesssim \lambda$, для чего следует сделать некоторые предположения о структуре волновой функции всей системы вихрей в целом.

Заметим, что при обходе каждого вихря системы по замкнутому контуру, не содержашему других вихрей, фаза $\phi$ волновой функции $\psi$ меняется на $\pm 2 \pi$ в зависимости от направления ("заряда") вихря, так что для изолированного вихря с центром в точке 0 волновая функция имеет вид

$$
\psi(\rho, \varphi, z)=f(r, z) e^{ \pm i \varphi}, \quad r=\sqrt{x^{2}+y^{2}}, \quad \operatorname{tg} \varphi=y / x .
$$

Здесь знак + или - в фазе волновой функции определяется направлением вихря. Это подсказывает нам вид волновой функции $f$ для системы $N_{+}$"положительных" и $N_{-}$ “отрицательных" вихрей, расположенных в точках $\left(x_{i}, y_{i}\right), 1 \leq i \leq\left(N_{+}+N_{-}\right)$:

$$
\psi(x, y, z)=\prod_{i=1}^{N_{+}} f\left(r_{i}, z\right) e^{i \varphi_{i}} \prod_{j=1}^{N_{-}} f\left(r_{j}, z\right) e^{i \varphi_{j}}
$$

где $r_{i}=\sqrt{\left(x-x_{i}\right)^{2}+\left(y-y_{i}\right)^{2}}-$ расстояние от точки $(x, y)$ до центра $\left(x_{i}, y_{i}\right) i$-го вихря, а $\varphi_{i}=s_{i} \operatorname{arctg}\left(\frac{y-y_{i}}{x-x_{i}}\right)$ - фаза волновой функции этого же вихря; $s_{i}$, как и раньше, "заряд" вихря. Ясно, что если расстояние между вихрями велико, то (7) переходит в суперпозиционное приближение, т.к. на величину тока $j(x, y, z) \sim|\psi(x, y, z)|^{2} \sum\left(\nabla \varphi_{i}+c \mathbf{a}_{i}\right)$ в окрестности вихря влияют в основном структура той функции $f\left(r_{i}, z\right)$, которая отлична от 1 в его окрестности, и соответствуюшая фаза $\varphi_{i}$. Подставляя $(7)$ в $(4)$ и постулируя некоторый вид функции $f(r, z)$, мы получим уравнение для а. Если решение этого уравнения подставить в выражение $(5)$ для $F_{\Gamma л}$, то мы получим выражение для энергии системы вихрей

$$
\begin{aligned}
& F_{\text {Гл }}=\int d \mathbf{r} d z\left[\xi^{2}|\nabla \psi(\mathbf{r}, z)|^{2}-\frac{\xi}{\sqrt{2}}|\psi(\mathbf{r}, z)|^{2} \mathbf{a}(\mathbf{r}, z) \times\right. \\
& \left.\times \sum_{i=1}^{N_{+}+N_{-}} \nabla \varphi_{i}+\frac{1}{2}\left(|\psi(\mathbf{r}, z)|^{2}-1\right)^{2}\right] .
\end{aligned}
$$

Выполнение этой программы связано с огромными техническими сложностями. Кроме того, выражение (8) необходимо представить (стандартным для статистической механики образом, см., например, [10]) как сумму одно-, двух- и т.д. частичных взаимодействий. В случае трехчастичных взаимодействий невозможно произвести для свободной энергии разложение в духе кольцевого приближения, а для учета трехчастичного взаимодействия по теории возмушений необходима трехчастичная функция распределения, для которой у нас нет сколь-нибудь разумной аппроксимации. Поэтому для учета только двухчастичных взаимодействий ограничимся в выражении (7) случаем $N_{+}=N_{-}=1$. Следует отметить, что даже в этом приближении задача о решении уравнений $(3),(4)$ остается чрезвычайно сложной и получаемый результат неприемлем для конкретных расчетов. В связи с этим при анализе уравнений ГЛ мы не будем стремиться к получению точной формы потенциала взаимодействия вихрей, а будем исследовать только основные черты его поведения при малых $r$. 
Заметим, что выражение (2) для потенциала по смыслу его вывода [7] справедливо только для $r \gg \lambda$. Нашей задачей являются снятие этого ограничения и внесение соответствуюших изменений в $\Phi_{i j}\left(r_{i j}\right)$.

Заменив решение уравнения (3) выражением (7) с $N_{+}=N_{-}=1$, мы будем исследовать уравнение (4) при заданном виде функции $\psi(\mathbf{r}, z)$. Оно в этом случае принимает вид

$$
\nabla^{2} \mathbf{a}=\mathbf{a}|\psi|^{2}-\sqrt{2} \xi|\psi|^{2}\left(\nabla \varphi_{1}+\nabla \varphi_{2}\right)
$$

Здесь $\varphi_{1}$ и $\varphi_{2}$ определены так же, как в (7). Это уравнение линейно по а, поэтому мы можем воспользоваться преобразованием Фурье

$$
\mathbf{a}(\mathbf{r})=\int \frac{d \mathbf{k} d q}{(2 \pi)^{3}} e^{i \mathbf{k r}+i q z} \mathbf{a}(\mathbf{k}, q),
$$

где $\mathbf{r}$ и $\mathbf{k}$ - двумерные векторы в плоскости $(x, y)$. Кроме того, вслед за Пирлом [7] будем предполагать, что пленка имеет достаточно малую толщину, чтобы можно было написать

$$
|\psi(\mathbf{r}, z)|^{2}=|\psi(\mathbf{r})|^{2} \delta(z) d=d \int \frac{d \mathbf{q} d k}{(2 \pi)^{3}} M(\mathbf{q}) e^{i \mathbf{q} \mathbf{r}+i k z}
$$

Для фурье-преобразования $\nabla \varphi$ воспользуемся следующим представлением [11]:

$$
\nabla \varphi_{i}=\operatorname{rot}\left(\hat{z} V_{i}(\mathbf{r})\right)
$$

где $\nabla^{2} V_{i}(\mathbf{r})=2 \pi s_{i} \delta\left(\mathbf{r}-\mathbf{r}_{i}\right)$. Тогда нетрудно получить преобразование Фурье для $V(\mathbf{r})$ и $\nabla \varphi(\mathbf{r})$ :

$$
\nabla \varphi_{i}(\mathbf{r})=\int \frac{d \mathbf{k}}{(2 \pi)^{2}} e^{i \mathbf{k}\left(\mathbf{r}-\mathbf{r}_{i}\right)} i[\mathbf{k} \times \hat{z}] \frac{2 \pi s_{i}}{k^{2}},
$$

где $r_{i}, \varphi_{i}, s_{i}$ - соответственно координаты центра, фаза и “заряд" $i$-го вихря, а $\hat{z}$ - единичньй орт в направлении $O z$. Выражение (13) следует понимать в смысле обобщенных функций. Соединяя воедино выражения (9), (10), (11), (13), получим

$$
\begin{array}{r}
\mathbf{a}(\mathbf{k})=\frac{d}{2 k} \int \frac{d \mathbf{k}^{\prime}}{(2 \pi)^{2}} M\left(\mathbf{k}^{\prime}\right)\left[\frac{2 \sqrt{2} \pi \xi}{\left|\mathbf{k}-\mathbf{k}^{\prime}\right|^{2}} \sum_{i=1,2} s_{i} e^{-i\left(\mathbf{k}-\mathbf{k}^{\prime}\right) \mathbf{r}_{i} \times}\right. \\
\left.\times i\left[\left(\mathbf{k}-\mathbf{k}^{\prime}\right) \times \hat{z}\right]-\mathbf{a}\left(\mathbf{k}-\mathbf{k}^{\prime}\right)\right] .
\end{array}
$$

Здесь введено обозначение

$$
\mathbf{a}(\mathbf{k})=\int \frac{d q}{2 \pi} \mathbf{a}(\mathbf{k}, q)
$$

Интегральное уравнение (14) эквивалентно уравнению (9). В пределе “точечных вихрей” $M(\mathbf{k})=(2 \pi)^{2} \delta(\mathbf{k})$ (что соответствует $|\psi(r)|^{2}=1$ ) и уравнение решается точно:

$$
\mathbf{a}_{0}(\mathbf{k})=\sum_{i=1,2} d \sqrt{2} \xi \frac{\pi i[\hat{k} \times \hat{z}]}{k(k+d / 2)} s_{i} e^{-i \mathbf{k} r_{i}}
$$


Подстановка этого решения в (8) с учетом того, что для $|\psi|^{2}=1$ имеет место $\nabla|\psi|=\left(|\psi|^{2}-1\right)=0$, дает выражение (2)для энергии взаимодействия вихрей, а после привлечения дополнительных соображений, необходимых для устранения расходимостей, связанных со сделанными приближениями (см. $[7,8])$, получим собственную энергию вихря. Отсюда становится ясно, что в приближении “точечных вихрей” справедливо суперпозиционное приближение, т.е. мы можем рассматривать магнитное поле каждого вихря независимо от других вихрей, а для вычисления энергии взаимодействия рассматривать энергию вихря в поле его соседа [7].

Для более точного учета структуры функции $|\psi(\mathbf{r})|^{2}$ положим

$$
|\psi(\mathbf{r})|^{2}=\prod_{i=1,2} f^{2}\left(\left|\mathbf{r}-\mathbf{r}_{i}\right|\right)
$$

где $f(\mathbf{r})$ - функция, описываюшая структуру отдельного вихря. Ее фурье-образ обозначим $m(\mathbf{k})$. Он связан с $M(\mathbf{k})$ следуюшим образом:

$$
M(\mathbf{k})=\int \frac{d \mathbf{k}^{\prime}}{(2 \pi)^{2}} m\left(\mathbf{k}-\mathbf{k}^{\prime}\right) m\left(\mathbf{k}^{\prime}\right) e^{-i \mathbf{k}^{\prime}\left(\mathbf{r}_{1}-\mathbf{r}_{2}\right)} e^{-i \mathbf{k} r_{2}}
$$

Заметим, что $f(r)$ мало отличается от 1 и для нее можно написать

$$
f^{2}(r)=1-h(r / \xi)
$$

так что

$$
m(\mathbf{k})=(2 \pi)^{2} \delta(\mathbf{k})-\int \frac{d \mathbf{r}}{\xi^{2}} \xi^{2} h(r / \xi) e^{-i(\mathbf{k} \xi)(\mathbf{r} / \xi)}=(2 \pi)^{2} \delta(\mathbf{k})-\xi^{2} \hat{h}(k \xi)
$$

Если решать уравнение (14) по теории возмушений, предполагая малость поправок, вносимых $h$, и считая нулевым приближением выражение (15) для $a_{0}$, то мы увидим, что выражение $a_{1}$ для следующего приближения будет пропорционально $d^{2}$ и в интересующем нас пределе $d \rightarrow 0$ несушественно по сравнению с $a_{0} \sim d$.

Подставим в свободную энергию (8) выражение (15) для $a_{0}$. Второе слагаемое в (8) принимает вид

$$
\begin{aligned}
F_{\text {ГЛ }}^{(2)}=-2 \pi^{2} d^{2} \xi^{2} \sum_{i, j=1,2} s_{i} s_{j} \int \frac{d \mathbf{k} d \mathbf{k}^{\prime}}{(2 \pi)^{4}} M(k) \times & \\
& \times \frac{\mathbf{k}^{\prime 2}+\left(\mathbf{k}^{\prime} \mathbf{k}\right)}{k^{\prime 2}\left(k^{\prime}+d / 2\right)\left|\mathbf{k}+\mathbf{k}^{\prime}\right|^{2}} e^{i \mathbf{k}^{\prime}\left(\mathbf{r}_{j}-\mathbf{r}_{i}\right)+i \mathbf{k} r_{j}} .
\end{aligned}
$$

Складывая это выражение с частью первого слагаемого, пропорциональной $(\nabla \varphi)^{2}, \mathrm{c}$ учетом (17) получим выражение для энергии двух вихрей с зарядами $s_{1}$ и $s_{2}$, центры которых находятся в точках $\mathbf{r}_{1}$ и $\mathbf{r}_{2}$, соответственно:

$$
F_{\Gamma л}=-2(2 \pi)^{2} \xi^{2} d \int \frac{d \mathbf{q}}{(2 \pi)^{2}} e^{i \mathbf{q}\left(\mathbf{r}_{2}-\mathbf{r}_{1}\right)} U(\mathbf{q})+F_{1},
$$


где

$$
\begin{aligned}
U(\mathbf{q})= & \int \frac{d \mathbf{k}_{1} d \mathbf{k}_{2}}{(2 \pi)^{4}}\left\{s_{1} s_{2} m\left(\mathbf{q}-\mathbf{k}_{1}\right) m\left(\mathbf{q}+\mathbf{k}_{2}\right)+m(\mathbf{q}) m\left(\mathbf{q}+\mathbf{k}_{1}+\mathbf{k}_{2}\right)\right\} \times \\
& \times \frac{\left(\mathbf{k}_{1} \mathbf{k}_{2}\right)}{k_{1}^{2} k_{2}^{2}} \frac{k_{1}}{k_{1}+d / 2},
\end{aligned}
$$

а $F_{1}$ - слагаемые в $F_{\Gamma л}$, зависящие от $\nabla|\psi|$ и $\left(|\psi|^{2}-1\right)^{2}$; эти слагаемые дадут вклад в энергию взаимодействия вихрей только на расстояниях $r \lesssim \xi$, так что, как будет ясно из дальнейшего, в нашем рассмотрении их можно опустить.

В пределе $m(\mathbf{k}) \sim \delta(\mathbf{k})$ выражение для $U(q)$ переходит в фурье-образ потенциала (2). Для получения поправок необходимо учесть высшие по $h$ члены.

3. Займемся теперь анализом выражения (22). После подстановки выражения (19) в $(22)$ получим, что $U(\mathbf{q})$ состоит из двух частей: знакопеременной части, пропорциональной $s_{1} s_{2}$, и знакопостоянного слагаемого, зависимость которого от расстояния между вихрями исчезает в пределе точечных вихрей. Это слагаемое является прообразом выражения для энергии изолированного вихря, однако в силу сделанных приближений оно расходится, и для получения энергии вихря необходимо использовать дополнительные соображения $[7,8]$.

Для анализа изменений, вносимых в зависящую от $r=\left|\mathbf{r}_{1}-\mathbf{r}_{2}\right|$ часть потенциала взаимодействия, следует рассмотреть в выражении для $U(q)$, получаюшемся при подстановке (19) в (22), слагаемые, в которых фигурирует $h(\xi q)$ и не появляется $\delta(q)$. Анализ этих слагаемых показывает, что изменения, вносимые в потенциал взаимодействия вихрей, меняют его поведение только на расстояниях $r \sim \xi$ (см. приложение).

Вместе с тем полученный в выражении (21) потенциал взаимодействия вихрей обладает рядом недостатков. Во-первых, для получения его явного вида нам необходимо сделать дополнительные предположения о виде функции $h(r / \xi)$, например

$$
h(r / \xi)=e^{-r^{2} / \xi^{2}} .
$$

Это не единственная возможная аппроксимация величины $h(r / \xi)$. Так, например, можно использовать выражение, предложенное Клемом [12],

$$
h(r / \xi)=\frac{1}{(r / \xi)^{2}+1} .
$$

Вторым недостатком является чрезвычайная сложность выражения (21), которая не позволяет провести с ним какие-либо расчеты (см. приложение).

По вышеупомянутым причинам мы не будем использовать конкретньй вид полученных выражений в дальнейшем. В то же время мы можем использовать полученные оценки как оправдание следующих соображений, позволяюших получить простое описание поведения потенциала взаимодействия вихрей на малых расстояниях.

Из (2) видно, что при приближении двух вихрей друг к другу энергия их взаимодействия стремится к бесконечности, знак которой зависит от “зарядов" $s_{i}$ и $s_{j}$ вихрей. Эта ситуация нефизична. На самом деле энергия взаимодействия двух "противоположно заряженных" вихрей должна иметь минимум при совпадении их центров. Это следует из того, что два “противоположно заряженных" вихря при совпадении их центров 
компенсируют друг друга, так что энергия такой системы равна 0. Отсюда очевидно, что для правильного потенциала $\tilde{\Phi}(r)$

$$
\tilde{\Phi}_{+-}(0)+2 \varepsilon_{1}=0,
$$

где $\varepsilon_{1}$ - собственная энергия вихря заряда 1 . Если рассматривать систему в отсутствие магнитного поля, то энергия вихря не зависит от его направления и равна $\left.{ }^{2}\right)$

$$
\varepsilon_{1}=\pi r_{0}^{2} \xi^{2} \frac{H_{c}^{2}}{8 \pi} d+\frac{\varphi_{0}^{2}}{16 \pi \Lambda}\left[H_{0}\left(\frac{\xi}{\Lambda}\right)-N_{0}\left(\frac{\xi}{\Lambda}\right)\right] .
$$

Здесь $H_{c}$ - термодинамическое критическое поле, $r_{0}$ - "радиус" вихря в единицах длины когерентности, величина порядка 1 . Радиус $r_{0}$ является вариационным параметром теории, характеризуюшим энергию ядра вихря. Как будет видно из дальнейшего, от его значения существенно зависит вид получаемых результатов.

Выражение (26) использовалось и в теории, развитой для точечных вихрей $[1,3]$. Однако оно устроено таким образом (см. приложение), что размеры вихря в нем учтены. Если учесть, что $\pi H_{c} \lambda \xi 2 \sqrt{2}=\varphi_{0}$, то получим для $\varepsilon_{1}$

$$
\varepsilon_{1}=\frac{\varphi_{0}^{2}}{16 \pi^{2} \Lambda}\left[r_{0}^{2} / 2+\pi\left(H_{0}\left(\frac{\xi}{\Lambda}\right)-N_{0}\left(\frac{\xi}{\Lambda}\right)\right)\right] .
$$

Аналогично для $\tilde{\Phi}_{++}\left(r_{i j}\right)$ и $\tilde{\Phi}_{--}\left(r_{i j}\right)$ следует положить

$$
\tilde{\Phi}_{ \pm \pm}(0)+2 \varepsilon_{1}=\varepsilon_{2} .
$$

Здесь $\varepsilon_{2}-$ энергия вихря заряда +2, которая, как и для обычного вихря, не зависит от его направления. Если дополнительно предположить, что

$$
\tilde{\Phi}_{+-}(0)=-\tilde{\Phi}_{++}(0),
$$

то получим, что $\varepsilon_{2}=4 \varepsilon_{1}$; это показывает, что образование одного вихря зарядом +2 энергетически менее выгодно, чем образование двух вихрей зарядом +1 [8].

Перейдем к модификации потенциала (2). Простейший способ сделать $\Phi_{+ \pm}(0)$ конечным - это представить, что взаимодействие вихрей локально, а их заряд "размазан" около центра вихря с плотностью $n(r)$, где $r$ - расстояние до центра вихря. В этом случае потенциал взаимодействия вихрей принимает вид

$$
\tilde{\Phi}_{i j}\left(\left|\mathbf{r}_{i}-\mathbf{r}_{j}\right|\right)=\int d \mathbf{r}^{\prime} \int d \mathbf{r}^{\prime \prime} n\left(\left|\mathbf{r}_{i}-\mathbf{r}^{\prime}\right|\right) \Phi_{i j}\left(\left|\mathbf{r}^{\prime}-\mathbf{r}^{\prime \prime}\right|\right) n\left(\left|\mathbf{r}^{\prime \prime}-\mathbf{r}_{j}\right|\right),
$$

где интегрирование идет по плоскости $\mathbf{r}=(x, y)$. При этом функция $n(r)$ отлична от нуля только в области $r \lesssim \xi$, что обеспечивает совпадение $\tilde{\Phi}_{i j}(r)$ и $\Phi_{i j}(r)$ при $r \gg \xi$ в соответствии с рассмотрением уравнений ГЛ (см. выше).

\footnotetext{
${ }^{2)}$ Начиная с этого момента мы возврашаемся к обычной системе единиц.
} 
Функция $\tilde{\Phi}_{i j}(r)$ отличается от $\Phi_{i j}(r)$ только при $r \lesssim \xi$ и удовлетворяет всем указанным выше требованиям к потенциалу взаимодействия вихрей. Кроме того, фурье-образы обоих потенциалов связаны простым соотношением

$$
\begin{aligned}
& \Phi_{q}=\int d \mathbf{r} e^{i \mathbf{q}^{r}} \Phi(\mathbf{r})=\frac{s_{i} s_{j} \varphi_{0}^{2}}{2 \pi \Lambda} \frac{1}{q(q+\alpha)}, \quad \alpha=1 / \Lambda \\
& \tilde{\Phi}_{q}=n_{q} \Phi_{q} n_{q}=\frac{s_{i} s_{j} \varphi_{0}^{2}}{2 \pi \Lambda} \frac{n_{q}^{2}}{q(q+\alpha)} .
\end{aligned}
$$

4. Теперь перейдем непосредственно к рассмотрению термодинамических характеристик системы. В работе [2] получено выражение для поправки к свободной энергии системы, обусловленной взаимодействием точечных вихрей (2):

$$
\beta \Delta F_{v}=\frac{1}{8 \pi^{2}} \int d \mathbf{q}\left\{\ln \left(1+\frac{\kappa^{2}}{q(q+\alpha)}\right)-\frac{\kappa^{2}}{q(q+\alpha)}\right\} .
$$

Здесь $\kappa^{2}=\frac{\beta \varphi^{2} \rho}{2 \pi \Lambda}, \beta=1 / k_{\mathrm{B}} T, \rho-$ плотность вихрей, а индекс $v$ обозначает, что свободная энергия берется в расчете на единицу двумерного объема. В случае потенциала (29) с учетом (31) можно получить

$$
\beta \Delta \tilde{F}_{v}=\frac{1}{8 \pi^{2}} \int d \mathbf{q}\left\{\ln \left(1+\frac{\kappa^{2} n_{q}{ }^{2}}{q(q+\alpha)}\right)-\frac{\kappa^{2} n_{q}{ }^{2}}{q(q+\alpha)}\right\} .
$$

Это выражение используется для получения температурной зависимости плотности свободных вихрей $\rho(T)$.

Выражение для поправки к свободной энергии в том виде, в каком оно записано в (33), непосредственно неприменимо, т.к. явный вид функции $n_{q}$ нам не известен. В качестве $n_{q}$ можно взять либо фурье-образ гауссова распределения, либо фурье-образ распределения (24). Однако оценки показывают, что вид получаемых результатов слабо зависит от конкретного вида $n_{q}[13,14]$. Для количественных расчетов выберем $n_{q}$ в простейшем виде:

$$
n_{q}= \begin{cases}1 & \text { при } q \leq B, \\ 0 & \text { при } q \geq B .\end{cases}
$$

Здесь $B$ - некоторая величина порядка $1 / \xi$, так что, как видно из выражения (29), на расстояниях $r \gg \xi$, т.е. при $q \ll 1 / \xi, n(r)$ можно считать дельта-функцией, и, следовательно, потенциал взаимодействия остается неизменным. На масштабе же $r \lesssim \xi$ потенциалы $\tilde{\Phi}(r)$ и $\Phi(r)$ сушественно различаются (см. выше). Для конкретных вычислений величину $B$ можно извлечь из критерия (25), откуда в пределе $\xi / \Lambda \rightarrow 0$ получается $B \approx 1 / \xi$, как и следовало ожидать.

Для $n_{q}$, взятой в виде $(34)$, легко получить из $(33)$

$$
\begin{aligned}
-16 \pi \Lambda^{2} \beta \Delta \tilde{F}_{v}= & (4 \zeta-1) J(B, \zeta)+(2 \zeta-1) \ln (\zeta)- \\
& -2 B^{2} \ln \left(1+\frac{\zeta}{B(B+1)}\right)-2(1-2 \zeta) \ln \left(\frac{B+1}{\sqrt{B^{2}+B+1}}\right),
\end{aligned}
$$


здесь $\zeta=\kappa^{2} \Lambda^{2}$, а

$$
J(B, \zeta)= \begin{cases}\frac{2}{\sqrt{4 \zeta-1}}\left\{\operatorname{arctg} \frac{2 B+1}{\sqrt{4 \zeta-1}}-\operatorname{arctg} \frac{1}{\sqrt{4 \zeta-1}}\right\} & \text { при } 4 \zeta-1 \geq 0, \\ \frac{1}{\sqrt{1-4 \zeta}} \ln \left[\left(\frac{2 B+1-\sqrt{1-4 \zeta}}{2 B+1+\sqrt{1-4 \zeta}}\right)\left(\frac{1+\sqrt{1-4 \zeta}}{1-\sqrt{1-4 \zeta}}\right)\right] & \text { при } 4 \zeta-1 \leq 0 .\end{cases}
$$

Эта функция является обобщением введенной в работе [2] функции $J(\zeta)$ и переходит в нее в пределе $B \rightarrow \infty$.

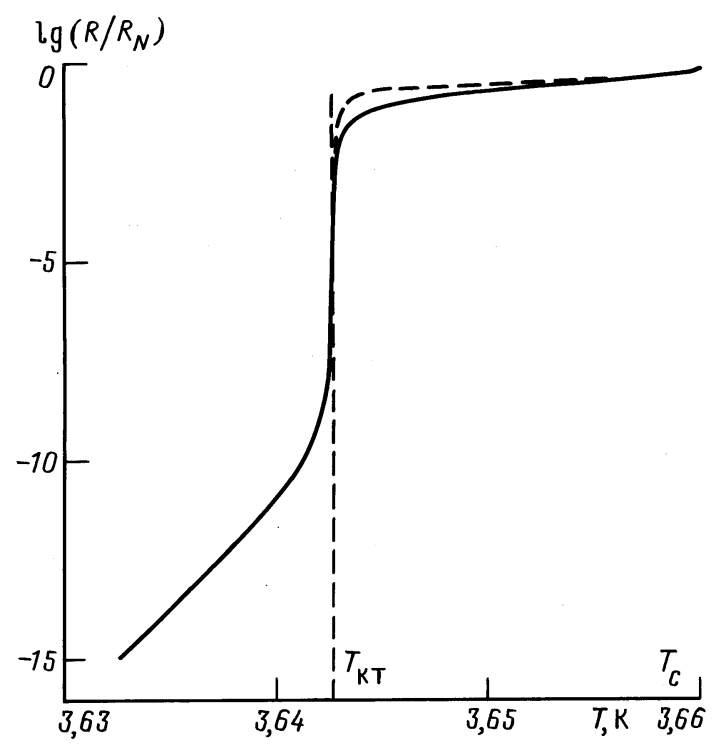

Рис. 1

Выражение для поправки к свободной энергии связано с уравнением для плотности $\rho$ вихрей (см., например, $[1,2,3])$ :

$$
\ln (\rho / 2 z)=-\frac{\partial \beta \Delta \tilde{F}_{v}}{\partial \rho}
$$

Диффференцируя выражение (35) по $\rho$, получим уравнение для плотности вихрей в отсутствие магнитного поля:

$$
\ln (\rho / 2 z)=\frac{\beta \varphi_{0}^{2}}{16 \pi^{2} \Lambda}\left\{\ln \left(\frac{(B+1)^{2}}{B^{2}+B+\zeta} \zeta\right)+J(B, \zeta)\right\}
$$

Выражение для $z$ мы считаем заданным (см. $[1,3])$. Заметим [3], что это же уравнение можно получить исходя из условия минимума по $\rho$ величины

$$
\frac{G(T)}{k_{\mathrm{B}} T \Omega}=\rho\left[\ln \left(\frac{\rho}{2 z}\right)-1\right]+\beta \Delta \tilde{F}_{v}
$$


а также непосредственно из вириального разложения [15]. В работах $[1,2,3]$ вместо уравнения (38) использовалось уравнение, в которое переходит (38) при $B \rightarrow \infty$,

$$
\ln (\rho / 2 z)=\frac{\beta \varphi_{0}^{2}}{16 \pi^{2} \Lambda}\{\ln (\zeta)+J(\zeta)\}
$$

Здесь $J(\zeta)$ - упомянутая выше функция, получаюшаяся из $(36)$ в пределе $B \rightarrow \infty$. С учетом формулы Бардина-Стефана мы можем связать решение этих уравнений с кривой сопротивления [16]:

$$
\frac{R}{R_{N}}=2 \pi \xi^{2} \rho
$$

5. Напомним, что уравнение (40), соответствуюшее пределу точечных вихрей, было численно решено в работах $[1,3]$. В работе [3] было обнаружено, что вид решения сильно зависит от величины "радиуса" $r_{0}$ вихря. Так, при $r_{0}$, большем некоторого критического значения $r_{0 c}$, зависимость $\rho(T)$ имеет резкую, хотя и без особенностей, зависимость от температуры $T$, а при $r_{0} \leq r_{0 c}$ решение уравнения (40) приобретает упоминавшиеся в разделе 1 нефизические свойства.

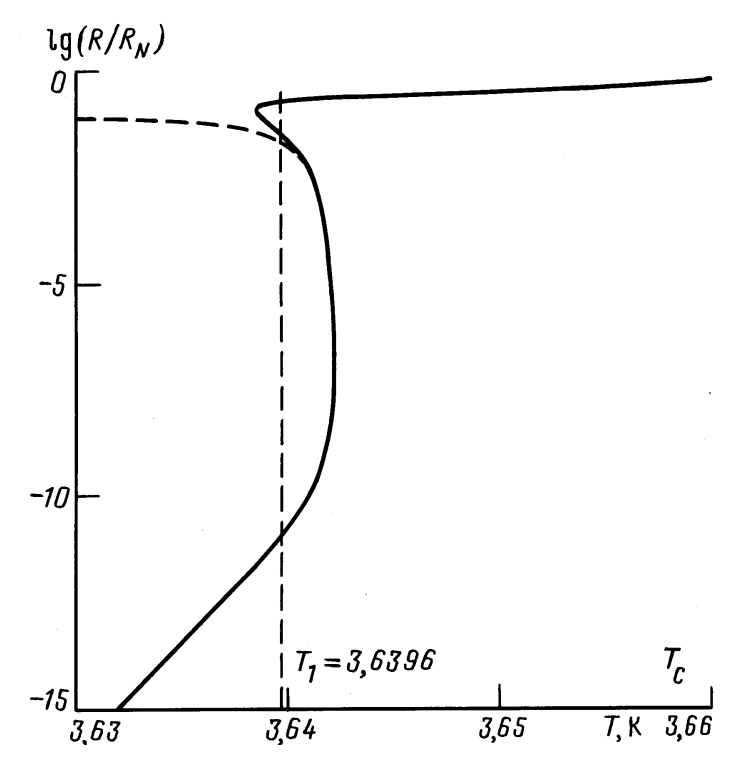

Рис. 2

На рис. 1 и 2 приведены решения уравнения (38) для "размытых" вихрей в случае $r_{0}=1,25$ и $r_{0}=0,9$, соответственно. Для сравнения штриховой линией показаны соответствуюшие решения "старого” уравнения (40).

Полученную зависимость $\rho$ от температуры естественно интерпретировать как увеличение с повышением температуры числа свободных вихрей в результате диссоциации пар вихрь-антивихрь. Для значений $r_{0}$, больших некоторого критического значения $r_{0}^{c}$, имеет место плавный, хотя и достаточно резкий переход (рис.1) от области с малым $\rho$ к области, где $\rho$ велико, подобный переходу КТ с отсутствием сингулярных зависимостей 
термодинамических величин от температуры. Такая ситуация схожа с той, что наблюдается в эксперименте на некоторых пленках [17]. Из рис. 1 видно, что учет конечности кора вихря при $r_{0}>r_{0}^{c}$ не приводит к качественным изменениям картины. Кривая лишь немного сдвигается, становясь более плавной в области больших плотностей.

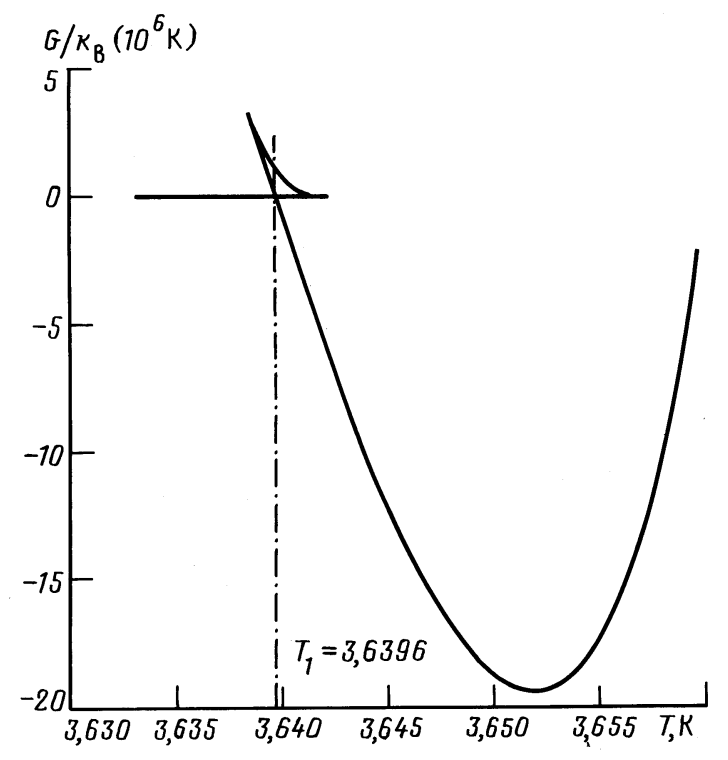

Рис. 3

Ситуация в корне меняется при $r_{0}<r_{0}^{c}$. В пренебрежении конечностью кора вихря (уравнение (40)) решение распадалось на две ветви, одна из которых приведена на рис. 2 штриховой линией. Подробно эти решения обсуждались в работе [3]. При учете конечности кора (уравнение (38)) две ветви заменяются одной, причем вид ее характерен для фазового перехода первого рода (сплошная линия на рис. 2). Температуру этого перехода можно получить из условия равенства свободных химических потенциалов $G$ (39). Зависимость потенциала $G$ от температуры вдоль решения уравнения (38) для $r_{0}=0,9$ показана на рис. 3 сплошной линией. Точка самопересечения этой кривой соответствует фазовому переходу первого рода (т.е. $G(T)$ имеет излом). Отметим, что при плавном переходе $\left(r_{0}<r_{0}^{c}\right)$ аналогичная кривая не имеет ни изломов, ни других сингулярностей (штриховая линия на рис. 3).

Заметим, что возможность диссоциации связанных пар (диполей) в двумерном кулоновском газе посредством перехода первого рода активно обсуждается в последнее время разными авторами (см., например, $[14,18,19,20,21]$ и ссылки в этих работах). Было обнаружено, что при малых активностях $z \sim e^{-\varepsilon_{1} / k_{\mathrm{B}} T}$ диссоциация происходит посредством перехода первого рода, в то время как при больших $z$ имеет место обычный переход КТ.

Отметим наконец, что при дальнейшем уменьшении $r_{0}$ решение уравнения $(38)$ все же “опрокидывается" и ведет себя подобно разрывным решениям уравнения (40) (см. [3]). Вероятно, следует более тщательно исследовать зависимость собственной энергии вихря от температуры и радиуса $r_{0}$ (см. также приложение). Кроме того, на форму пере- 
хода может оказать влияние конкретный выбор функции $n_{q}$, а также два важных физических явления, которые мы до сих пор не учитьвали: пиннинг вихрей случайными примесями и экранировка потенциала связанными парами вихрей.

Все авторы благодарят за поддержку Российский фонд фундаментальных исследований и Международный научный фонд (гранты No. 94-02-03415, No. MUN000 и No. MUN300).

\section{Приложение}

Рассмотрим выражение (22). С учетом (19) его можно переписать в виде

$$
U(q)=\frac{-s_{1} s_{2}}{q(q+d / 2)}+s_{1} s_{2} \Delta_{1} U(q)+\Delta_{2} U(q)
$$

где $s_{1} s_{2} \Delta_{1} U(q)$ и $\Delta_{2} U(q)$ - соответственно знакопеременная и знакопостоянная поправки к потенциалу взаимодействия:

$$
\begin{aligned}
\Delta_{1} U(q)= & \int \frac{d \mathbf{k}}{(2 \pi)^{2}} \frac{\mathbf{q} k}{q^{2} k^{2}} \frac{k}{k+d / 2} \xi^{2} \hat{h}((\mathbf{q}-\mathbf{k}) \xi)- \\
& -\int \frac{d \mathbf{k}}{(2 \pi)^{2}} \frac{\mathbf{q} k}{q^{2} k^{2}} \frac{q}{q+d / 2} \xi^{2} \hat{h}((\mathbf{k}+\mathbf{q}) \xi)+ \\
& +\int \frac{d \mathbf{k} d \mathbf{k}^{\prime}}{(2 \pi)^{4}} \frac{\mathbf{k} k^{\prime}}{k^{2} k^{\prime 2}} \frac{k}{k+d / 2} \xi^{4} \hat{h}((\mathbf{q}-\mathbf{k}) \xi) \hat{h}\left(\left(\mathbf{q}+\mathbf{k}^{\prime}\right) \xi\right), \\
\Delta_{2} U(q)= & \delta(\mathbf{q}) \tilde{\varepsilon}(q)+\xi^{2} \hat{h}(\mathbf{q} \xi) \int \frac{d \mathbf{k}}{(2 \pi)^{2}} \frac{\mathbf{k}(\mathbf{q}+\mathbf{k})}{k^{2}(\mathbf{k}+\mathbf{q})^{2}} \frac{k}{k+d / 2}+ \\
& +\xi^{2} \hat{h}(\mathbf{q} \xi) \int \frac{d \mathbf{k} d \mathbf{k}^{\prime}}{(2 \pi)^{4}} \frac{\mathbf{k} k^{\prime}}{k^{2} k^{\prime 2}} \frac{k}{k+d / 2} \xi^{2} \hat{h}\left(\left(\mathbf{q}+\mathbf{k}+\mathbf{k}^{\prime}\right) \xi\right) .
\end{aligned}
$$

Как нетрудно заметить, для энергии взаимодействия вихрей нам важны только те слагаемые в $U(q)$, которые не пропорциональны $\delta(\mathbf{q})$. Поэтому нам не важен явньй вид величины $\tilde{\varepsilon}(q)$. Следует заметить, что т.к. в выражении для $\mathbf{a}(\mathbf{r}, z)$ мы ограничились первым приближением (см. (15)), то выражение $\tilde{\varepsilon}(q)$, как было отмечено выше, расходится.

Согласно выражению (П.1) энергия пары вихрей, включающая в себя и их собственную энергию, пропорциональна величине

$$
\begin{aligned}
G(r) & =G_{0}(r)+\int \frac{d \mathbf{q}}{(2 \pi)^{2}} e^{i \mathbf{r q}}\left(s_{1} s_{2} \Delta_{1} U(\mathbf{q})+\Delta_{2} U(\mathbf{q})\right)=G(r)^{\prime}+\Delta G(r), \\
G_{0}(r) & =-\int \frac{d \mathbf{q}}{(2 \pi)^{2}} e^{i \mathbf{r q}} \frac{s_{1} s_{2}}{q(q+d / 2)}
\end{aligned}
$$

причем энергия $\Phi$ взаимодействия вихрей должна быть определена таким образом, чтобы $\Phi(r) \rightarrow 0$ при $r \rightarrow \infty$. Не обрашаюшаяся в 0 на бесконечности часть выражения (П.4) даст вклад в энергию вихря $\varepsilon_{1}$. Рассмотрим в (П.4) слагаемые, порождаемые поправками к основному выражению для $U(q)$ в (П.1). Для этого в (П.2) и (П.3) совершим замену переменных:

$$
\mathbf{q} \xi=\mathbf{x}, \quad \mathbf{k} \xi=\mathbf{y}, \quad \mathbf{k}^{\prime} \xi=\mathbf{y}^{\prime}
$$


Теперь выражения (П.2) и (П.3) можно записать в виде

$$
\Delta_{1} U(q)=\xi^{2} A(x), \quad \Delta_{2} U(q)=\delta(\mathbf{q}) \tilde{\varepsilon}(q)+\xi^{2} B(x),
$$

где $A(x)$ и $B(x)$ - некоторые функции аргумента $\mathbf{q} \xi$, явный вид которых нам не сушественен. Подставляя их в выражение (П.4), получим, что $\Delta G$ является функцией от $r / \xi$, так что при $r \gg \xi$ (напомним, что нас интересуют масштабы $\xi \ll r \ll \lambda$ ) ее можно считать постоянной и равной $\Delta G(r)=\Delta G(\infty)$. По указанной выше причине она не будет изменять потенциал взаимодействия вихрей на расстояниях $r \gg \xi$. Таким образом, мы приходим к выводу, что учет конечного радиуса вихря изменяет потенциал парного взаимодействия только на масштабе $r \lesssim \xi$.

Для иллюстрации приведенных аргументов рассмотрим выражение для $\Delta_{1} U$ в случае $(23)$ :

$$
\hat{h}(q \xi)=\pi e^{-q^{2} \xi^{2} / 4}
$$

Подставляя это выражение в $\Delta_{1} U(q)$, после несложных выкладок получим (см. (П.5))

$$
\Delta_{1} U(q)=\xi^{2}\left[\frac{1}{x} L_{1}(x)+\frac{1}{x+d \xi / 2} L_{2}(x)-L_{1}(x) L_{2}(x)\right],
$$

где

$$
\begin{aligned}
& L_{1}(x)=\frac{1}{2} \int_{0}^{\infty} d y \frac{y}{y+d \xi / 2} I_{1}\left(\frac{x y}{2}\right) e^{-y^{2} / 4} e^{-x^{2} / 4}, \\
& L_{2}(x)=\frac{1}{2} \int_{0}^{\infty} d y I_{1}\left(\frac{x y}{2}\right) e^{-y^{2} / 4} e^{-x^{2} / 4},
\end{aligned}
$$

а $I_{1}(x)$ - функция Инфельда (модифицированная функция Бесселя). Как легко видеть, выражение (П.6) имеет слишком громоздкий вид, не позволяющий использовать его в конкретных расчетах.

Отметим, что в проделанных выкладках мы опускали всюду члены, дающие вклад в собственную энергию вихрей. Кроме того, в рамках использованного приближения (см.(15), (7)) часть этих слагаемых расходится. Поэтому приведенные выше выражения не могут служить основой для улучшения оценки величины $\varepsilon_{1}$. Вместе с тем в выражении (26) для энергии вихря второе слагаемое (пропорциональное $H_{0}(\xi / \Lambda)-N_{0}(\xi / \Lambda)$ ) отвечает за энергию магнитного поля, а в первом слагаемом учтена энергия кора. Это означает, что для наилучшей оценки $\varepsilon_{1}$ необходимо рассматривать $r_{0}$ как вариационный параметр, выбираемый для наилучшего совпадения полученных результатов с экспериментальными. Возможно также, что иным выбором аналогичного параметра можно устранить оставшиеся нефизические решения.

\section{Список литературы}

[1] Ryzhov V.N., Tareyeva E.E. // Phys. Rev. B. 1993. V. 48. №17. P. 12907-12911.

[2] Рыжсов B.Н., Тареева E.E. // ТМФ. 1993. Т. 96. № 3. С. 425-437.

[3] Ryzhov V.N., Tareyeva E.E. // Phys. Rev. B. 1994. V. 49. №9. P. 6162-6173.

[4] Kosterlitz J.M., Thouless D.J. // J. Phys. C. 1973. V. 6. P. 1181. 
[5] Kosterlitz J.M. // J. Phys. C. 1974. V. 7. P. 1046.

[6] Ирз Д.Ю., Рыжов В.Н., Тареева Е.Е. // ТМФ. 1995. Т. 104. № 2. С. 337-347.

[7] Pearl J. // Low Temperature Physics LT9 / Eds. J.G. Daunt, D.O. Edwards, F.J. Milford, M. Yagub. New York: Plenum, 1965. P. 566.

[8] Де Женн П. Сверхпроводимость металлов и сплавов. М.: Мир, 1968.

[9] Лифииц Е.М., Питаевский Л.П. Статистическая физика (Часть 2). М.: Наука, 1978.

[10] Балеску Р. Равновесная и неравновесная статистическая механика. М.: Мир, 1978.

[11] Minnhagen P., Nylén M. // Phys. Rev. B. 1985. V. 31. P. 5768.

[12] Clem J.R. // Inhomogeneous Superconductors - 1979 (Berkeley Springs WV), Proceedings of the Conference on Inhomogeneous Superconductors, AIP Conf.Proc. No. 58 / Eds. D.U.Gubser, T.L.Francavilla, S.A.Wolf, J.R.Leibowitz. New York: AIP, 1979. P. 245.

[13] Cataudella V., Minnhagen P., Weber H. // J. Phys: Condens. Matter. 1990. V. 2. P. 2345.

[14] Xu B.-W., Zhang Yu.-M. // Phys. Rev B. 1994. V. 50. P. 18651.

[15] Майер Дж., Гепперт-Майер М. Статистическая механика. М.: Мир, 1980.

[16] Тинкхам M. Введение в сверхпроводимость. М.: Атомиздат, 1980.

[17] Hsu J.W.P., Kapitulnik A. // Phys.Rev. B. 1992. V. 45. №9. P. 4819.

[18] Minnhagen P. // Rev. Mod. Phys. 1987. V. 59. P. 1001.

[19] Minnhagen P., Wallin M. // Phys. Rev. B. 1989. V. 40. P. 5109.

[20] Lee J.-R., Teitel S. // Phys. Rev. Lett. 1991. V. 66. P. 2100.

[21] Jonsson A., Minnhagen P., Nylen M. // Phys. Rev. Lett. 1993. V. 70. P. 1327.

Институт физики высоких давлений

Российской академии наук

Поступила в редакцию 28.VI.1995 г.

\section{Yu. Irz, V.N. Ryzhov, E. E. Tareeva \\ THE STATISTICAL MECHANICS OF VORTEX SYSTEM IN THIN SUPERCONDUCTING FILM IN CYCLE APPROXIMATION. III. FINITE SIZE VORTEX CORE EFFECTS}

The statistical mechanics of vortex system in two-dimensional superconductor is discussed in the frame of the cycle approximation. The finite size of vortex core and vortex core shape are taken into account. This effect leads to the crossover of the vortex-antivortex pair unbinding transition from the usual continuous Kosterlitz-Thouless-like behavior for large core energies to the first order transition for low energies. 